\title{
GROUP RINGS WITH FINITE CENTRAL ENDOMORPHISM DIMENSION
}

\author{
by B. A. F. WEHRFRITZ
}

(Received 9 March, 1982)

1. Introduction. Let $F$ be any field. Denote by $\mathfrak{X}_{F}$ the class of all groups $G$ such that every irreducible $F G$-module has finite dimension over $F$ and by $3_{F}$ the class of all groups $G$ such that every irreducible $F G$-module has finite dimension over its endomorphism ring. Clearly $\mathfrak{X}_{F} \subseteq \mathfrak{B}_{F}$.

The study of the classes $\mathfrak{X}_{F}$ arose out of work of P. Hall and later Roseblade on residual finiteness of certain soluble groups. Recently $[\mathbf{2}, \mathbf{5}, \mathbf{7}, \mathbf{8}$ and 9$]$ the soluble $\mathfrak{X}_{F}$-groups have been almost completely described. The classes $3_{F}$ arise in connection with injective modules [3, Sections 3.2 and 12.4]. In some unpublished work [1] B. Hartley has effectively described all locally finite $3_{F}$-groups and, coupled with Section 3 of [7], this also describes all locally finite $\mathfrak{X}_{F}$-groups. It seems likely that a successful assault on soluble $3_{F}$-groups will require considerably more knowledge of soluble 'linear' groups over division algebras than the present author has. We therefore suggest the following intermediate class.

Let $\bigvee_{F}$ be the class of all groups $G$ such that every irreducible $F G$-module has finite dimension over the centre of its endomorphism ring. By a theorem of Kaplansky [3, 5.3.4 and 5.1.6] this is the class of all groups $G$ such that every primitive image of $F G$ satisfies a polynomial identity. Much of the work on $\mathfrak{X}_{F}$ goes through with suitable modifications for $\mathfrak{Y}_{F}$ and the object of this note is to indicate these modifications.

It is convenient in places to use the algebra of group classes. As usual $\mathfrak{F}, \mathfrak{S}, \mathfrak{A}, \mathfrak{P}$ and $\subseteq$ denote respectively the classes of finite, finitely generated, abelian, polycyclic and soluble groups and $\mathrm{S}, \mathrm{Q}, \mathrm{L}$ and $\mathrm{R}$ the subgroup, quotient, local and residual operators. If $p$ is a prime, $\mathfrak{B}_{\mathrm{p}}$ is the class of all groups with a series of finite length whose factors are cyclic or Prüfer $p^{\infty}$-groups. Throughout $u$ will denote the characteristic of the field $F$ (so that $u \geqslant 0)$. If $G$ is a group and $p$ a prime then $O_{0}(G)=\langle 1\rangle$ and $O_{p}(G)$ is the maximal normal $p$-subgroup of $G$. Also $\Lambda(G)$ is the subgroup of elements $g$ of $G$ such that for every finite subset $X$ of $G$, the $F C$-centre of $\langle g, X\rangle$ contains $g$.

THEOREM 1. Let $F$ be any field. Then

$$
\text { (S) } \cap \mathfrak{S}_{\mathfrak{F}} \cap \mathfrak{V}_{F}= \begin{cases}\mathfrak{S} \cap \mathfrak{A} \mathfrak{F} & \text { if } F \text { is not locally finite, } \\ \mathfrak{B} \mathfrak{F} & \text { if } F \text { is locally finite. }\end{cases}
$$

THEOREM 2. If $F$ is a field of characteristic $u \geqslant 0$ that is not locally finite, then $\mathfrak{S}_{\mathfrak{F}} \cap \mathfrak{V}_{F}$ is the class of all groups $B$ with

$$
G / O_{u}(G) \in \mathfrak{H} \mathfrak{F}, O_{u}(G) \subseteq \Lambda(G) \text { and } O_{u}(G) \in \mathfrak{S} .
$$

Our information about soluble $\mathfrak{V}_{F}$-groups for $\mathfrak{\mho}$ locally finite is even less complete than about soluble $\mathfrak{X}_{F}$-groups.

Glasgow Math. J. 24 (1983) 169-176. 
THEOREM 3. Let $F$ be a locally finite field of characteristic $u$ and let $G$ be $a$ soluble-by-finite $\mathfrak{Y}_{F}$-group. Then $G$ has normal subgroups $O_{u}(G) \subseteq B \subseteq H \subseteq N \subseteq G$ satisfying:

(a) $O_{u}(G) \subseteq \Lambda(G)$ and is $O_{u}(G)$ soluble;

(b) $B / O_{u}(G)$ is periodic abelian, $G / C_{G}\left(B / O_{u}(G)\right)$ is periodic, $B$ is the maximal periodic normal subgroup of $H$ and has finite index in the maximal periodic normal subgroup of $G$;

(c) $H / B$ is a torsion-free by finite $\mathfrak{B}_{u}$-group;

(d) $N / H$ is abelian with no elements of order $u$;

(e) $G / N$ is finite.

By way of comparison consider the following. Let $F$ be a locally finite field and let $G=A \times H$ where $A$ is abelian and $H$ polycyclic. By [7, 2.2 and 3.1] and [9, Theorem 3] we have that $G \in \mathfrak{X}_{F}$ if and only if $A \in \mathfrak{X}_{F}$, and the latter implies that $A$ has finite torsion-free rank.

Proposition. With $F$ and $G$ as above, $G \in \mathfrak{V}_{F}$ if and only if either $A$ has finite torsion-free rank or $H$ is abelian-by-finite.

Thus although Theorems 1 and 2 above are strikingly similar to their $\mathfrak{X}_{F}$ counterparts, it would seem that characterizations of $\widetilde{S} \cap \mathfrak{X}_{F}$ and $\mathfrak{S} \cap \mathfrak{V}_{F}$ for $F$ locally finite will have to differ noticeably. A further difference is that if $F$ and $K$ are fields with $F \leqslant K$ then $\mathfrak{Y}_{F} \supseteq \mathfrak{V}_{K}$ (an easy result-but see below), while this is not usually true for $\mathfrak{X}_{F}$ and $\mathfrak{X}_{K}$. Indeed there is a tendency for the reverse to be true. For example $\mathfrak{A} \cap \mathfrak{X}_{F} \subseteq \mathfrak{U} \cap \mathfrak{X}_{K}$ always, and thus $\mathfrak{S} \cap \mathfrak{X}_{F} \subseteq \subseteq \cap \mathfrak{X}_{K}$ whenever $F$ is not locally finite.

\section{Preliminary remarks.}

2.1. $\mathfrak{X}_{F} \subseteq \mathfrak{Y}_{F} \subseteq \mathfrak{B}_{F}$ for any $F$.

2.2. $\mathfrak{A} \subseteq \mathfrak{V}_{F}$ for any $F$.

\section{3. $\mathfrak{V}_{\mathrm{F}}$ is $\langle\mathrm{s}, \mathrm{Q}\rangle$-closed for any $\mathrm{F}$.}

The quotient closure of $\mathfrak{V}_{F}$ is trivial. Let $H$ be a subgroup of the $\mathfrak{Y}_{F}$-group $G$ and let $W$ be an irreducible $F H$-module. By Hall's lemma $[7,2.1]$ there is an irreducible $F G$-module $V$ containing $W$ as an $F H$-submodule. Now $F H / \mathrm{Ann}_{F H} W$ is an image of $F H / A n n_{F H} V$, which is isomorphic to a subalgebra of $F G / A n n_{F G} V$. By hypothesis this satisfies a standard polynomial identity; whence $F H / A n_{F H} W$ does too.

2.4. $G \in \mathfrak{Y}_{F}$ if and only if $G /(G \cap(1+J(F G))) \in \mathfrak{Y}_{F}$. In particular $G \in \mathfrak{Y}_{F}$ if $G /\left(O_{u}(G) \cap \Lambda(G)\right) \in \mathfrak{V}_{F}$.

Here $J(F G)$ is the Jacobson radical of $F G$. The first part is immediate and the second follows since $\left(O_{u}(G) \cap \Lambda(G)\right)-1$ generates a nil ideal of $F G$. Also immediate from the definitions is the following.

2.5. $G /(G \cap(1+J(F G)))$ is residually an irreducible linear group (over various extension fields of $F$, namely the centres of the endomorphism rings of the irreducible FGmodules).

2.6. $G \cap(1+J(F G)) \subseteq O_{u}(G)$ with equality if $G \in \mathfrak{V}_{F}$. 
The containment is well known and is recorded in $[\mathbf{7}, 2.5]$. Suppose $G \in \mathfrak{Y}_{F}$. Let $\rho$ be an irreducible representation of $G$, finite dimensional over some extension field of $F$. Then $O_{u}(G \rho)$, being unipotent and completely reducible $[6,9.1 \mathrm{v}$ and 1.8$]$, is trivial. Thus $O_{u}(G) \subseteq \bigcap$ ker $\rho$, which is $G \cap(1+J(F G))$ by 2.5 .

2.7. If $H$ is a subgroup of the group $G$ of finite index then $H \in \mathfrak{V}_{F}$ if and only if $G \in \mathfrak{Y}_{F}$.

If $G \in \mathfrak{V}_{F}$ then $H \in \mathfrak{V}_{F}$ by 2.3. Suppose $H \in \mathfrak{V}_{F}$. Again by 2.3 we may assume that $H$ is normal in $G$. Let $V$ be an irreducible $F G$-module, set $R=F G /$ Ann $_{F G} V$ and let $S$ be the natural image of $F H$ in $R$. By a version of Clifford's theorem [3, 7.2.16] $V$ is a direct sum of a finite number of irreducible $F H$-modules. Since $H \in \mathfrak{V}_{F}$ it follows that the centre $Z$ of $S$ is a direct sum of a finite number of fields and that $S$ is finitely generated as $Z$-module. Clearly $G$ normalizes $Z$ and $H$ centralizes $Z$. Thus the finite group $G / H$ acts on $Z$. By a result from invariant theory (in fact an easy extension of a result from Galois theory) $Z$ can be generated as $C_{Z}(G)$-module by $|G / H|$ elements. Since $C_{Z}(G)$ is central in $R$ the result follows.

As a companion to 2.7 we have the following.

2.8. Let $F, K$ be fields with $F \leqslant K$. Then $\mathfrak{V}_{F} \supseteq \mathfrak{V}_{K}$ with equality if $(K: F)$ is finite.

Let $G \in \mathfrak{V}_{K}$ and suppose that $V$ is an irreducible $F G$-module. Then $V \cong F G / A$ for some right ideal $A$. Now $A K \neq K G$ so that $A$ lies in a maximal right ideal $B$ of $K G$. Clearly $A=B \cap F G$ and $V$ embeds into the irreducible $K G$-module $W=K G / B$. Since $K G / A n_{K G} W$ satisfies a standard polynomial identity, so does $F G / A n n_{F G} W$ and hence $F G / A n_{F G} V$. Therefore $G \in \mathfrak{V}_{F}$.

Now let $G \in \mathfrak{Y}_{F}$ where $(K: F)$ is finite. Let $W$ be an irreducible $K G$-module. Then $W$ is finitely $F G$-generated, so $W$ contains a maximal $F G$-submodule $V$. Now $W A n n_{F G}(W / V)$ is a $K G$-submodule of $W$ in $V$ and so is zero. Thus $A n n_{F G}(W / V)=$ $F G \cap A n_{K G} W$. By hypothesis $F G / A n_{F G}(W / V)$ satisfies a polynomial identity. Hence so does $K G / \mathrm{Ann}_{K G} W=K\left(\left(F G+\mathrm{Ann}_{K G} W\right) / \mathrm{Ann}_{K G} W\right)$, for example by $[3,5.1 .3]$.

2.9 (B. Hartley). $\mathrm{L} \mathfrak{F} \cap \mathfrak{V}_{F}$ is the class of locally finite groups $G$ with $G / O_{u}(G)$ abelianby-finite.

Hartley's theorem [1] is that $G / O_{u}(G)$ is abelian-by-finite if merely $G \in \mathrm{L} \widetilde{F} \cap \oiint_{F}$. The converse follows from $2.2,2.7$ and 2.4 .

2.10. The wreath product $G=(\mathbb{Z} / n \mathbb{Z}) \mathrm{wr} \mathbb{Z}$ is not in $3_{F}\left(\right.$ and hence not in $\left.\mathfrak{Y}_{F}\right)$ for $n=0$, $2,3, \ldots$

By 2.3 we may assume that $n$ is a prime power. Then by $[3,9.2 .8]$ the group ring $F G$ is primitive and not simple, but if $G \in \mathcal{B}_{F}$ then every primitive image of $G$ is simple by the density theorem.

2.11. If $G \in \mathfrak{Y}_{F}$ then $G / O_{u}(G) \in \mathrm{LR} \mathfrak{F} \cap \mathrm{R}(\mathfrak{A} \mathfrak{F}) \subseteq \mathfrak{A}$. R $\mathfrak{F}$.

We may assume that $O_{u}(G)=\langle 1\rangle$. Linear groups are in LR $\mathfrak{F}[6,4.2]$ and $G$ is residually linear by 2.5 and 2.6 . Thus $G \in \mathrm{LR} \mathfrak{F}$. Let $\rho$ be a finite-dimensional irreducible representation of $G$ over some extension field of $F$. Clearly $G$ can contain no non-cyclic 
free subgroups. If $u=0$ then $G \rho$ is soluble-by-finite by Tits' theorem $[6,10.17]$. If $u \neq 0$ Tits' theorem yields only that $G \rho \in \mathcal{S}_{\mathrm{L}} \mathfrak{F}$. But then 2.9 with 2.3 and $[6,3.8]$ implies that here also $G \rho$ is soluble-by-finite. Since $\rho$ is irreducible, in both cases $G \rho$ is abelian-byfinite by Mal'cev's theorem $[6,3.5]$. This proves that $G \in \mathbf{R}(\mathfrak{A} \mathfrak{F})$ and clearly $\mathbf{R}(\mathfrak{A} \mathfrak{F}) \subseteq \mathfrak{A R} \mathfrak{F}$.

3. Finitely generated groups. Write $\tau(G)$ for the maximal periodic normal subgroup of a group $G$.

3.1. Let $G$ be a soluble-by-finite $\mathfrak{V}_{F}$-group, where $F$ is not locally finite. Then $G / \tau(G)$ is abelian-by-finite.

If $G$ is countable we can repeat the proof of $[7,5.1]$ (except that the representations are now over extension fields of $F$ ). A standard argument reduces the general case to the countable case (cf. the proof of $[8$, Lemma 1]).

3.2. Let $G$ be a finitely generated $\mathfrak{V}_{F^{-}}$group. If $A$ is a periodic abelian section of $G$ (and $u$-free if $u>0$ ) then $A$ has finite exponent.

Suppose otherwise. By [8, Lemma 2] there is an infinite image of $A$ of rank 1 . Hence we may assume that $A$ has rank 1 . But then there is an irreducible $F A$-module that is faithful on $A$. Thus by Hall's lemma there is a finite-dimensional irreducible representation $\rho$ of $G$ over some extension field of $F$ whose kernel avoids $A$. Tits's and Mal'cev's theorems yield that $G \rho$ is abelian-by-finite. Also $G$ is finitely generated and $A$ is periodic. Thus $A$, being isomorphic to a section of $G \rho$, is finite. This contradiction completes the proof.

3.3. Let $x \in \mathbb{C}^{*}$ be such that, for some prime $u, x$ is integral over $\mathbb{Z}[1 / u]$ but not over $\mathbb{Z}$. Let $A=\mathbb{Z}\left[x, x^{-1}\right] \subseteq \mathbb{C}$. Multiplication by $x$ is an automorphism of $A$; so we may let $G$ be the split extension $\langle x\rangle[A$ of $A$ by $\langle x\rangle$. If $F$ is any field of characteristic $u$ then $G$ is not a $\mathfrak{Y}_{F}$ group.

We already know $[7,4.5]$ that $G$ is not an $\mathfrak{X}_{F}$-group. In the proof of that result we constructed a certain irreducible $F G$-module $V$ where

$$
V=\bigcup_{i \in \mathbb{Z}} U_{i}, \quad U_{i} \cong_{F A} F A /\left(A_{i}-1\right) F A \cong F\left(A / A_{i}\right) \quad \text { and } \quad A_{i}=\sum_{j \leqslant i} \mathbb{Z} x^{i} \subseteq A
$$

Let $K$ be the kernel of the representation of $G$ on $V$. Then $A \cap K \subseteq \bigcap_{i} A_{i}=\{0\}$. Thus $[A, K]=\langle 1\rangle$ and yet $\langle x\rangle$ acts faithfully on $A$. Therefore $K=\langle 1\rangle$. If $G \in Y_{F}$ then Mal'cev's theorem yields that $G \cong G / K$ has an abelian normal subgroup $B$ of finite index $m$ say. Then $x^{m}$ acts trivially on $A \cap B$, which is impossible since $x$ has infinite order, $A \cap B$ is nontrivial and $A$ is a domain. Consequently $G \notin Y_{F}$.

We remark in passing that the above proof contains the following.

3.4. If $G$ and $F$ are as in 3.3 then $F G$ is primitive.

3.5. Proof of Theorem 1. In view of 3.1 it suffices to prove that if $G \in \circlearrowleft \cap \mathfrak{S}_{\mathfrak{F}} \cap \mathfrak{V}_{F}$ then $G$ is polycyclic-by-finite. By the usual reductions (using passage to a subgroup of 
finite index, induction on derived length and the maximal condition on normal subgroups) we may assume that $G$ has a non-trivial abelian normal subgroup $A$ that is either of prime exponent or is torsion-free, and that every proper image of $G$ is polycyclic.

Suppose $A$ has prime exponent $q$. In view of 2.3 and 2.10 there is no subgroup of $G$ isomorphic to $(\mathbb{Z} / q \mathbb{Z})$ wr $\mathbb{Z}$. Now $A$ is finitely $G$-generated, $G / A$ is polycyclic and every proper image of $\mathbb{F}_{q}\left[X, X^{-1}\right]$ is finite. It follows that $A$ is finite, so that $G$ is polycyclic in this case as required. (I have lifted this trick from [2].)

Now assume that $A$ is torsion-free. Trivially $A \cap \tau(G)=\langle 1\rangle$, so that if $F$ is not locally finite then $G$ is abelian-by-finite by 3.1 (applied twice). Suppose $F$ is locally finite. By a lemma of $P$. Hall $\left[4,9.53\right.$ Corollary 1] $A$ contains a free abelian subgroup $A_{0}$ such that $A / A_{0}$ is periodic with finite spectrum. If $q$ is a prime not in the spectrum of $A / A_{0}$ then $A_{0} \cap A^{q}=A_{0}^{q}$. Now $G / A^{q}$ is polycyclic. Hence $A_{0} / A_{0}^{q}$ is finite and $A$ has a finite rank. Let $A_{1} / A_{0}=O_{u^{\prime}}\left(A / A_{0}\right)$, so $A / A_{1}$ is a $u$-group and $A_{1} / A_{0}$ a $u^{\prime}$-group. $A_{1} / A_{0}$ has finite exponent by 3.2 and $A_{1}$ is torsion-free. Thus $A_{1}$ is free abelian of finite rank.

$G$, being soluble of finite rank, is nilpotent-by-abelian-by-finite [4, 3.25]. Repeating if necessary our initial reductions we may assume that $G^{\prime}$ centralizes $A$. Now we choose $A$ of minimal rank. Thus $\mathbb{Q} \otimes_{\mathbb{Z}} A$ is irreducible as $\mathbb{Q} G$-module and by Schur's lemma there is a finite field extension $K$ of $\mathbb{Q}$, an embedding $\lambda: A \rightarrow K$ and a homomorphism $\mu: G \rightarrow K^{*}$ such that the $G$-module structure of $A$ is induced by multiplication of $A \lambda$ by $G \mu$ in $K$. Since $A / A_{1}$ is a $u$-group we have $A \lambda \subseteq A_{1} \lambda \mathbb{Z}[1 / u]$ and so $G \mu$ is integral over $\mathbb{Z}[1 / u]$.

Suppose $G \mu$ is integral over $\mathbb{Z}$. Then $\mathbb{Z}[G \mu]$ is finitely generated as $\mathbb{Z}$-module and consequently so is $A_{1} \lambda \mathbb{Z}[G \mu]$. Thus $\left\langle A_{1}^{G}\right\rangle$ is a finitely generated (abelian) group. Since $A_{1} \neq\langle 1\rangle$ the group $G /\left\langle A_{1}^{G}\right\rangle$ is polycyclic and therefore so is $G$. We are left with the case where $G$ contains an element $g$ such that $g \mu$ is not integral over $\mathbb{Z}$. Necessarily $g \mu$ has infinite order. Let $a \in A \backslash\langle 1\rangle$. Then $\langle\mathrm{g}, a\rangle \in \mathfrak{V}_{F}$ by 2.3 and yet

$$
\langle g, a\rangle=\langle g\rangle\left\langle a^{\langle g\rangle}\right\rangle \cong\langle g \mu\rangle\left[a \lambda \mathbb { Z } [ g \mu , g ^ { - 1 } \mu ] \cong \langle g \mu \rangle \left[\mathbb{Z}\left[g \mu, g^{-1} \mu\right] .\right.\right.
$$

This contradicts 3.3 and completes the proof of Theorem 1 .

\section{Soluble groups.}

4.1. Let $F$ be a field (with $\operatorname{char} F=u \geq 0$ as always) and $G=\langle x\rangle[A$ be the split extension of its abelian normal subgroup $A$ of finite torsion-free rank by the infinite cyclic group $\langle x\rangle$. Suppose that $A \backslash\langle 1\rangle$ contains no elements of order $u$, and that if $F$ is locally finite then $A$ is periodic. If $G \in \mathfrak{Y}_{F}$ then $C_{\langle x\rangle}(A) \neq\langle 1\rangle$.

This generalizes [8, Lemma 4 and $\mathbf{9}$, Lemma 1] and the proof of 4.1 is similar to these. Consider the proof of Lemma 4 of [8]. In the second and third paragraphs of that proof we construct certain direct sums of finitely many irreducible representations of $G$. In the context of 4.1 they are no longer finite dimensional over $F$ but are finite-dimensional over suitable extension fields of $F$. The results of [6] still apply and the construction of $X$ goes through as in [8].

In paragraphs 4, 5 and 6 of the proof we constructed an extension field $K$ of $F$, an irreducible $K G$-module $V$ and a maximal $F G$-submodule $W$ of $V$. Recall that we were 
seeking a contradiction. Let $\sigma$ be the representation of $G$ on $V$ and $\tau$ of $G$ on $V / W$. Since $G \in \mathfrak{Y}_{F}$ the group $G \tau$ is an irreducible linear group over some extension of $F$. By [8, Lemma 3] there is a normal subgroup $L$ of $G$ of finite index and containing $A$. ker $\tau$ such that $L \tau$ is abelian. Now $V((\operatorname{ker} \tau)-1)$ is a $K G$-submodule of $V$ in $W$ and therefore is zero. Thus ker $\sigma=\operatorname{ker} \tau$. Let $m=(G: L)$. Then $\left[x^{m}, A\right] \subseteq \operatorname{ker} \sigma$, which implies (see the construction of $V$ ) that $a \phi=a^{x^{m}} \phi$ for every $a \in A$. This is false by construction.

4.2. Let $G$ be a $\mathfrak{V}_{F}$-group with $O_{u}(G)=\langle 1\rangle$. Then $G$ has a normal subgroup $K$ with $G / K u$-free-abelian by finite and $\tau(K)$ abelian and of finite index in $\tau(G)$.

By Hartley's theorem (2.9) and $[3,12.1 .2]$ there exists an abelian characteristic subgroup $T$ of $\tau(G)$ of finite index. Let $Q$ be a maximal $u$-subgroup of $\tau(G)$. Since $O_{u}(G)=\langle 1\rangle$ the subgroup $T$ is a $u^{\prime}$-group and $Q$ is finite. By 2.11 there exists $K_{1}$ normal in $G$ with $G / K_{1}$ abelian-by-finite and $Q \cap K_{1}=\langle 1\rangle$. Also by 2.11 there exists $K_{2}$ normal in $G$ with $G / K_{2}$ abelian-by-finite, $T \subseteq K_{2}$ and $\left(\tau(G) \cap K_{2}\right) / T=O_{u}(G / T)$. Set $K_{0}=K_{1} \cap K_{2}$. Clearly $G / K_{0}$ is abelian-by-finite, and $\tau\left(K_{0}\right)=K_{0} \cap \tau(G)=K_{0} \cap T$ by elementary Sylow theory. If $X$ is any irreducible linear group over an extension field of $F$ then $O_{u}(X)=\langle 1\rangle$. Thus the proof of 2.11 shows that we can choose $K_{0}$ with $O_{u}\left(G / K_{0}\right)=\langle 1\rangle$. Finally set $K=T K_{0}$.

4.3. Let $G$ be a soluble-by-finite $\mathfrak{Y}_{F^{-}}$group where $F$ is not locally finite. Then $G / O_{u}(G)$ is abelian-by-finite.

This can be proved along the lines of the proof of [8, Theorem 2]. However we indicate a better approach using ideas from both [5] and [8].

By [8, Lemma 1] we may assume that $G$ is countable. Also we may assume that $O_{u}(G)=\langle 1\rangle$. Choose $K$ as in 4.2. Also $G / \tau(G)$ is abelian-by-finite by 3.1. Hence $G$ has a normal subgroup $H$ of finite index with $H^{\prime} \subseteq K \cap \tau(G)$. Thus $H^{\prime}$ is periodic abelian. Also $O_{u}(H)=\langle 1\rangle$.

Let $x_{1}, x_{2}, \ldots$ be a transversal of $H^{\prime}$ to $H$. Suppose we have constructed $r_{1}, \ldots, r_{i-1}>0$ such that $A_{i}=\left\langle x_{i}^{r_{i}}: j<i\right\rangle H^{\prime}$ is abelian. Since $A_{i}$ is normal in $H$ we have $O_{u}\left(A_{i}\right)=\langle 1\rangle$ and 4.1 yields the existence of $r_{i}>0$ such that $\left[A_{i}, x_{i}^{r_{i}}\right]=\langle 1\rangle$ (if $\left|x_{i}\right|<\infty$ set $r_{i}=\left|x_{i}\right|$ ). Then $A_{i+1}=A_{i}\left\langle x_{i}^{r}\right\rangle$ is abelian. By induction we construct an abelian normal subgroup $A=\bigcup_{i \geq 1} A_{i}$ containing $H^{\prime}$ with $H / A$ periodic.

Let $a \in A$. We claim that $[a, H]$ has finite exponent $e(a)$ say. For, if not, by [8, Lemma 2] there is an infinite-rank-1 image of $[a, H] ;[a, H]$ is contained in $H^{\prime}$ and is periodic. By Hall's lemma there is an irreducible representation $\rho$ of $H$ over some extension of $F$ such that $[a, H] \rho$ is infinite. But $\left(H \rho: C_{H_{\rho}}(A \rho)\right)$ is finite, e.g. by $[\mathbf{8}$, Lemma $3]$, so $[a, H] \rho=\left\langle a^{-1} a^{H}\right\rangle \rho$ is finitely generated, abelian and periodic. This contradiction confirms the existence of finite $e(a)$.

Let $Q$ be a maximal torsion-free subgroup of $A$ and set $B=\left\langle a^{e(a)}: a \in Q\right\rangle$. Now $a \in A$ stabilizes the series $H \supseteq[a, H] \supseteq\langle 1\rangle$ since $A \supseteq[a, H]$ is abelian. Thus $a^{e(a)}$ centralizes $H$ and in particular $B$ is normal in $H$. Also $H / B$ is periodic, so by 2.9 again $H / B$ has a normal subgroup $N / B$ of finite index such that $N^{\prime} B / B$ is a $u$-group. But $N^{\prime} \subseteq H^{\prime}$ is a $u^{\prime}$-group, so that $N^{\prime} \subseteq B \cap H^{\prime}=\langle 1\rangle$ since $B \subseteq Q$ is torsion-free. The proof of 4.3 is complete. 
4.4. Proof of Theorem 2. Let $G \in \mathfrak{S} \mathfrak{F} \cap \mathfrak{Y}_{F}$. Then $G / O_{u}(G) \in \mathfrak{A} \mathfrak{F}$ by $4.3, O_{u}(G) \subseteq$ $\Lambda(G)$ by 2.6 and $[7,2.9]$, and $O_{u}(G)$ is clearly soluble.

Conversely suppose $G$ is a group with $G / O_{u}(G) \in \mathfrak{A} \mathfrak{F}$ and $O_{u}(G) \subseteq \Lambda(G)$. Then $G / O_{u}(G) \in \mathfrak{V}_{F}$ by 2.2 and 2.7 , so that $G \in \mathfrak{V}_{F}$ by 2.4 . If also $O_{u}(G)$ is soluble then $G \in \widetilde{\varsigma}$.

4.5. Proof of Theorem 3. By 2.6 and $[7,2.9]$ we have $O_{u}(G) \subseteq \Lambda(G)$ and $O_{u}(G)$ clearly is soluble. From now on assume that $O_{u}(G)=\langle 1\rangle$. By 4.2 there exist normal subgroups $B \subseteq H_{1} \subseteq N_{1}$ of $G$ with $\left(G: N_{1}\right)$ finite, $N_{1} / H_{1}$ abelian and $u$-free, $B=\tau\left(H_{1}\right)$ an abelian $u^{\prime}$-group and $(\tau(G): B)$ finite. $G / C_{G}(B)$ is periodic by 4 .1. Since $G \in \widetilde{S} \widetilde{F}$ we may choose $N_{1}$ to be soluble.

Let $X$ be a free abelian section of $N_{1} / B$ of infinite rank. There exists a purely transcendental extension $K$ of $F$ for which there is a homomorphism of $F X$ onto $K$ that is one-to-one on $X$. Hence by Hall's lemma there is an irreducible representation $\rho$ of $G / B$ over some extension of $F$ such that $\operatorname{ker} \rho$ avoids $X$. Also $G \rho$ is abelian-by-finite with $O_{u}(G \rho)=\langle 1\rangle$. Apply this to a free abelian subgroup of maximal rank in each factor with infinite torsion-free rank of the derived series of $N_{1} / B$. It follows that $G$ contains normal subgroups $N_{2} \subseteq N_{1}$ and $H_{2} \subseteq H_{1} \cap N_{2}$ with $\left(G: N_{2}\right)$ finite, $N_{2} / H_{2}$ abelian and $u$-free, $B \subseteq \mathrm{H}_{2}$ and $\mathrm{H}_{2} / \mathrm{B}$ is poly-(abelian with finite torsion-free rank). By a theorem of Mal'cev [4, 9.34 and 9.39.3] and the finiteness of $(\tau(G): B)$, the section $\mathrm{H}_{2} / B$ is torsion-free by finite, and soluble of finite rank.

Now let $X$ be a periodic abelian $u^{\prime}$-section of $H_{2} / B$ of rank 1 . Then $X$ can be embedded into the multiplicative group of the algebraic closure of $F$ so that by Hall's lemma applied to the extension of $F$ generated by this image of $X$ there exists an irreducible representation $\rho$ of $G$ over some extension of $F$ such that $\operatorname{ker} \rho$ avoids $X$. Again $G \rho$ is abelian-by-finite with $O_{u}(G \rho)=\langle 1\rangle$. Hence we can find normal subgroups $N \subseteq N_{2}$ and $H \subseteq H_{2} \cap N$ with $(G: N)$ finite, $N / H$ abelian and $u$-free, $B \subseteq H$ and $H / B \in \mathfrak{B}_{u}$. The proof is complete.

4.6. Proof of Proposition. Let $G \in \mathfrak{V}_{F}$ and suppose that $A$ has a free abelian subgroup $X$ of infinite rank. If $x$ is an indeterminate there is a homomorphism of $X$ onto $F(x)^{*}$, which can be extended to a homomorphism $\phi$ of $A$ into $\overline{F(x)}$ * by injectivity, the bar here denoting the algebraic closure. Then $K=F[A \phi]$ is a field that is not locally finite. Let $V$ be any irreducible $K H$-module. Then $\phi$ extends to a homomorphism of $F G$ onto $K H$ and $V$ becomes an irreducible $F G$-module with $\operatorname{End}_{F G} V=\operatorname{End}_{K H} V$. Since $G \in \mathfrak{V}_{F}$ these endomorphism rings are finite dimensional over their centres and we have $H \in \mathfrak{B} \cap \mathfrak{V}_{K}$. Thus $H$ is abelian-by-finite by Theorem 1 .

Conversely if $H$ is abelian-by-finite, so is $G$, and $G \in \mathfrak{V}_{F}$ by 2.2 and 2.7. Now suppose that $A$ has finite torsion-free rank. Let $\rho$ be an irreducible representation of $F G$ and set $J=(F A) \rho$. As in the proof of [9, Theorem 3], the ring $J$ is a field. If $X$ is a free abelian subgroup of $A$ of maximal rank, then $J$ is integral over $(F X) \rho$, so that the latter too is a field. But $X$ is finitely generated and thus $(F X) \rho$ is a finite extension of $F$ by the Nullstellensatz. Consequently $J$ is locally finite. Now $(F G) \rho$ is a homomorphic image of $J H$ and by Roseblade's theorem $[3,12.3 .7] J H \in \mathfrak{X}_{F}$. Therefore $(F G) \rho$ is finite dimensional over its central subfield $J$ and we have $G \in \mathfrak{V}_{F}$ as required. 


\section{REFERENCES}

1. B. Hartley, preprint.

2. I. M. Musson, Representation of infinite soluble groups, preprint.

3. D. S. Passman, The Algebraic Structure of Group Rings (John Wiley \& Sons, 1977).

4. D. J. S. Robinson, Finiteness Conditions and Generalized Soluble Groups (2 vols) (SpringerVerlag, 1972).

5. R. L. Snider, Solvable groups whose irreducible modules are finite dimensional, Comm. Algebra 10 (1982), 1477-1485.

6. B. A. F. Wehrfritz, Infinite Linear Groups (Springer-Verlag, 1973).

7. B. A. F. Wehrfritz, Groups whose irreducible representations have finite degree, Math. Proc. Cambridge Philos. Soc. 90 (1981), 411-421.

8. B. A. F. Wehrfritz, Groups whose irreducible representations have finite degree II, Proc. Edinburgh Math. Soc., 25 (1982), 237-243.

9. B. A. F. Wehrfritz, Groups whose irreducible representations have finite degree III, Math. Proc. Cambridge Philos. Soc. 91 (1982), 397-406.

Queen Mary College,

LONDON,

ENGLAND.

E1 4NS 